\title{
The risk factors involved in airway mucus plug in children with ADV pneumonia
}

\author{
Jing-jing Huang ${ }^{1}$, Lin Yuan ${ }^{1}$, Zhi-qiang Zhuo ${ }^{1}$, Ming-zhen $\mathrm{Li}^{1}$, and xingdong wu ${ }^{1}$ \\ ${ }^{1}$ xiamen children's hospital
}

February 2, 2022

\begin{abstract}
Background: The risk factors for mucus plug in children with adenovirus (ADV) pneumonia. Methods and materials:A retrospective analysis was conducted of children diagnosed ADV pneumonia and underwent bronchoscopy. The patients were divided into a mucus plug group (39 cases) and a non-mucus plug group (53 cases). The children's data including sex, age, clinical presentation, laboratory test parameters, imaging and bronchoscopic data were collected. The risk factors for the development of airway mucus plug were analysed by multifactorial logistic regression. Results: There were no statistically significant differences in sex, age, fever, hospitalization days, mixed infection, WBC count, neutrophil proportion, CRP, and D-dimer (all P $>0.05$ ); Thermal range, calcineurin (PCT), lactate dehydrogenase (LDH), Pleural effusion and associated decreased breath sounds was significantly higher in mucus plug group than in non-mucus plug group; multifactorial logistic regression analysis showed that the duration of fever, $\mathrm{PCT}$, and $\mathrm{LDH}$ were independent risk factors for the formation of mucus plugs. The critical values of ROC curves were pyroprocedure [?]6.5 d, PCT [?]0.705 ng/ml and LDH [?]478.5 U/L. Conclusion: Duration of fever, PCT and $\mathrm{LDH}$ levels were the independent risk factors for the formation of an airway mucus plug in children with ADV pneumonia.
\end{abstract}

\section{Hosted file}

airway mucus plug in children with adv pneumonia_.doc available at https://authorea.com/ users/458585/articles/555099-the-risk-factors-involved-in-airway-mucus-plug-in-childrenwith-adv-pneumonia

\section{Hosted file}

figure.docx available at https://authorea.com/users/458585/articles/555099-the-risk-factorsinvolved-in-airway-mucus-plug-in-children-with-adv-pneumonia 